\title{
Effects of scent enrichment on behavioral and physiological indicators of stress in zoo primates
}

\author{
Stefano Vaglio ${ }^{1,2}$ (1) | Stefano S. K. Kaburu ${ }^{3}$ (1) | Richard Pearce ${ }^{1}$ | Luke Bryant $^{1}$ | \\ Ailie McAuley $^{1}$ | Alexandria Lott ${ }^{1}$ | Demi J. Sheppard ${ }^{1}$ | Sarah Smith ${ }^{1}$ | \\ Beth E. Tompkins $^{1}$ | Emily J. Elwell ${ }^{1}$ | Sara Fontani ${ }^{1}$ (b) | \\ Christopher H. Young ${ }^{1}$ ( ) | Giovanna Marliani ${ }^{4}$ | Pier Attilio Accorsi ${ }^{4}$
}

${ }^{1}$ Department of Biology, Chemistry and Forensic Science, University of Wolverhampton, Wolverhampton, UK

${ }^{2}$ Department of Anthropology, Behaviour \& Ecology and Evolution Research (BEER)

Centre, Durham University, Durham, UK

${ }^{3}$ Department of Biomedical Science \& Physiology, University of Wolverhampton, Wolverhampton, UK

${ }^{4}$ Dipartimento di Scienze Mediche Veterinarie, Università di Bologna, Bologna, Italy

\section{Correspondence}

Stefano Vaglio, Department of Biology, Chemistry \& Forensic Science, University of Wolverhampton, Wulfruna St.,

Wolverhampton WV1 1LY, United Kingdom. Email: S.Vaglio@wlv.ac.uk

\section{Funding information}

Primate Society of Great Britain (PSGB), Grant/Award Number: Captive Care Grant - round 2018

\begin{abstract}
Captive breeding is vital for primate conservation, with modern zoos serving a crucial role in breeding populations of threatened species and educating the general public. However, captive populations can experience welfare issues that may also undermine their reproductive success. To enhance the wellbeing of endangered zoo primates, we conducted a study to assess the effects of a new scent enrichment program on captive red-ruffed lemurs (Varecia rubra), black howler monkeys (Alouatta caraya), siamangs (Symphalangus syndactylus), lar gibbons (Hylobates lar) and orangutans (Pongo pygmaeus pygmaeus). We combined behavioral observations and fecal endocrinology analyses to evaluate the effects of a series of essential oils (benzoin, lavender, lemongrass) on five captive troops $(N=19)$ housed at Dudley Zoo \& Castle and Twycross Zoo (UK). We recorded observations of natural speciesspecific and abnormal stress-related behaviors for $480 \mathrm{~h}$ using instantaneous scan sampling. We collected 189 fecal samples and measured the fecal cortisol concentrations using radioimmunoassay. We found a significant effect of the scent enrichment on behaviors, with red-ruffed lemurs and black howler monkeys reducing their social interactions, as well as red-ruffed lemurs and lar gibbons decreasing their stress-related behaviors after they were exposed to the series of essential oils. We also found that red-ruffed lemurs displayed a significant increase in fecal glucocorticoids following exposure to essential oils. Our contradictory findings suggest that the effects of this series of essential oils may change depending on the speciesspecific social lives and olfactory repertoires of primates. In conclusion, we cannot recommend using these essential oils widely with zoo primates without additional evaluation.
\end{abstract}

\section{KEYWORDS}

essential oils, stereotypic behaviors, social interactions, fecal cortisol

This is an open access article under the terms of the Creative Commons Attribution License, which permits use, distribution and reproduction in any medium, provided the original work is properly cited.

(c) 2021 The Authors. American Journal of Primatology Published by Wiley Periodicals LLC 


\section{1 | INTRODUCTION}

Almost half of the total primate species recognized today worldwide are classified as endangered or critically endangered in the wild, primarily due to human activities (Estrada et al., 2017). Therefore, raising global scientific and public awareness of the plight of the world's primates is now vital (Estrada et al., 2017). Zoos may play a major role (Mellor et al., 2015) as zoo animal populations are usually managed to educate the public regarding wildlife and their habitats and to preserve endangered species through captive breeding and reintroduction programs (Schulte-Hostedde \& Mastromonaco, 2015). However, captive populations, potentially serving as buffers against extinction, experience problems that impair them from being viable for reintroduction into the wild. More specifically, zoo animal populations face reproductive challenges which have so far inhibited them from serving as viable "reserve populations" (Meier, 2016). Additionally, managing zoo populations is challenging because of the mismatch between natural and captive environments and the knockon effects this has on the repertoire of behaviors exhibited (Carroll et al., 2014). Primates have evolved distinct behavioral patterns and difficulty in engaging in these behaviors can cause frustration or boredom, which, in turn, can lead to stress and development of abnormal behaviors (G. R. Hosey, 2005) that may undermine their individual welfare and ultimately their breeding success.

To maintain captive healthy populations modern zoos take part in conservation breeding programs. As reproductive success is linked to how closely captive environmental conditions mirror those that primates would be experiencing in the wild (Meier, 2016), zoos also use environmental enrichments to manage captive populations. Environmental enrichment and conservation breeding programs are directly related, as enrichment is a dynamic iterative process that changes an animal's environment, increasing its behavioral choices and prompting a wider range of natural and species-specific behaviors and abilities (Ben-Ari, 2001). Furthermore, enrichment can contribute to promoting resiliency to stress, which helps animals recovering from adverse stimuli (Quirke \& O'Riordan, 2011), improving both the exhibit from the visitor perspective and the reproductive performance of the hosted animals (Carlstead \& Shepherdson, 1994). Enrichment can also foster the essential skills that animals need for their survival if reintroduced into their habitat (Danial Rioldi, 2013).

Scent-based enrichments can be effective at increasing active behaviors in zoo animals and improve their welfare (Fay \& Miller, 2015; Quirke \& O'Riordan, 2011; Samuelson et al., 2017). However, this is not always the case and some authors reported findings that are less clear or indicate that scent enrichment has little effect (Myles \& Montrose, 2015; Wells et al., 2007). The delivery mechanism of the scent and the type of scents used are crucial for the implementation of novel olfactory enrichment programs (Baker et al., 2018). The majority of studies have used spices or essential oils rather than focusing on natural or biological scents, but this may not necessarily be appropriate for all species (Wells et al., 2007). The main goal of olfactory enrichment is to improve the welfare of animals in captive environments, but there is also the possibility that the use of scents can have additional positive impacts. For example, scents may elicit both behavioral and physiological responses and therefore the use of olfactory enrichment can be potentially used to promote beneficial impacts on reproductive success (Rafacz \& Santymire, 2014).

Primates are traditionally considered "microsmatic" (i.e., with a reduced olfactory sense; Negus, 1958) and, as many uses of enrichment are ad hoc and unrecorded, only a small proportion of formal studies on olfactory enrichment has been undertaken on primate species (Clark \& King, 2008). However, various lines of evidence suggest that chemical communication may be important in primates (Setchell et al., 2010). In particular, it has become increasingly clear that the sense of smell plays a crucial role in primate sociosexual communication, with semiochemicals (i.e., behavior- and physiology-modifying chemicals; Norland \& Lewis, 1976) being important for kin recognition, mate choice and the regulation of sociosexual behaviors (Vaglio et al., 2016). However, little is known about the overall effects of olfactory enrichment on primate species.

The overarching aim of our work is to design and test a new scent enrichment program to enhance the well-being of critically endangered zoo primates. In this context, we carried out a preliminary study which aimed to assess the effects of a series of essential oils (namely, benzoin, lavender, and lemongrass) on behavioral and physiological indicators of stress in five captive primate species: Red-ruffed lemurs (Varecia rubra), black howler monkeys (Alouatta caraya), siamangs (Symphalangus syndactylus), lar gibbons (Hylobates lar), and orangutans (Pongo pygmaeus pygmaeus). As the majority of studies of scent enrichment on zoo primates focus on essential oils, spices or herbs (Wells et al., 2007), we chose three essential oils due to their ecological relevance to non-human primates (benzoin; e.g., Horvath et al., 2007), effectiveness in domestic animals and humans (lavender; reviewed in Wells, 2009), and efficacy in sheltered cats and dogs as well as in zoo-housed exotic animals (lemongrass; e.g., Ellis \& Wells, 2010; Holland, 2018; Wells, 2004). The primate species investigated in this study are currently classified as critically endangered (red-ruffed lemurs, orangutans), endangered (lar gibbons, siamangs), or threatened (black howler monkeys) largely due to the deforestation, logging, and hunting activities that threaten the habitat and survival of these species across their ranges (IUCN, 2020). Therefore, designing and implementing strategies that improve the welfare and breeding success of these species in captivity is particularly crucial.

In this study, we predicted that the scent enrichment would reduce the stress levels of zoo primates, which would be reflected in significant changes in behavioral (i.e., increase of the frequency of social behaviors, and decrease of the frequency of stress-related behaviors) and physiological (i.e., decrease of fecal glucocorticoid concentrations or FGCs) indicators of well-being when comparing before (i.e., baseline period) and after (i.e., post enrichment period) the scent enrichment program. Particularly, this should occur in relatively "macrosmatic" primates (i.e., primate species with greater levels of olfactory function; Smith \& Bhatnagar, 2004) such as lemurs. 


\section{2 | MATERIAL AND METHODS}

\section{1 | Study subjects and housing}

We studied five captive troops of red-ruffed lemurs, black howler monkeys, siamangs, lar gibbons, and orangutans housed at Dudley Zoo \& Castle (red-ruffed lemurs, lar gibbons, orangutans) and Twycross Zoo (black howler monkeys, siamangs) in the United Kingdom. The troop of red-ruffed lemurs $(N=3)$ consisted of two related adult males (brothers; both aged 15 years at the beginning of the study period) and one unrelated adult female (aged 14 years). The troop of black howler monkeys $(N=5)$ was a family group and consisted of one adult female (aged 13 years) and her offspring-one juvenile female (aged 5 years) and three juvenile males (aged 4, 4, and 3 years). The troop of siamangs $(N=3)$ was a family group and consisted of one adult male (aged 14 years), one adult female (aged 14 years), and their son-one juvenile male (aged 5 years and 6 months). The troop of lar gibbons $(N=5)$ was a family group and consisted of one adult male (aged 16 years), one adult female (aged 17 years) and their offspring-one juvenile female (aged 6 years) and two young males (aged 2 years and 6 months). The troop of orangutans $(\mathrm{N}=3)$ was a family group and consisted of one adult male (aged 21 years), one adult female (aged 24 years), and their daughter-one juvenile female (aged 5 years).

We carried out behavioral observations and fecal sampling from July to September in 2016-2019 (Table 1). All troops lived in indoor enclosures (heated to $28^{\circ} \mathrm{C}$ ) with access to outdoor enclosures (i.e., "visitor walkthrough" enclosure in the case of red-ruffed lemurs).

\section{2 | Study protocol}

We divided the overall study period into three periods: Baseline, scent enrichment, post enrichment. We collected behavioral data and fecal samples for 2-6 days per study period (10 days in total), 2 days per week over a 3-month period (1-week baseline; 3-week scent enrichment, i.e., benzoin, lavender, and lemongrass; 1-week post enrichment), for each species (Table 1) to use a combination of both behavioral (e.g., naturalistic species-specific behaviors, stereotypic behaviors) and physiological (e.g., corticosteroid levels)

TABLE 1 Study protocol

\begin{tabular}{|c|c|c|}
\hline Species & Site & Period of time \\
\hline Red-ruffed lemurs & Dudley Zoo & July-September 2019 \\
\hline Black howler monkeys & Twycross Zoo & $\begin{array}{l}\text { July-September } 2016 \\
\text { July-September } 2019\end{array}$ \\
\hline Siamangs & Twycross Zoo & July-September 2016 \\
\hline Lar gibbons & Dudley Zoo & $\begin{array}{l}\text { July-September } 2018 \\
\text { July-September } 2016\end{array}$ \\
\hline Orangutans & Dudley Zoo & $\begin{array}{l}\text { July-September } 2018 \\
\text { July-September } 2019\end{array}$ \\
\hline
\end{tabular}

Note: All experimental protocols included a 2-day baseline, 6-day scent enrichment, and 2-day post enrichment. methods to assess the effects of scent enrichment (See Sections 2.2.2 and 2.2.3).

\subsection{1 | Scent enrichment}

We cut white cotton sheets into 75-cm-long and 5-cm-wide strips, which were soaked with 20 drops Naissance $100 \%$ pure essential oil diluted with $12 \mathrm{ml}$ of cold boiled water. We prepared the scent cotton strips during the early morning of each sampling day over the scent enrichment period. We positioned these strips around both indoor and outdoor enclosures; focusing on the outdoor enclosure, we tied them approximately $1 \mathrm{~m}$ from the ground around the climbing frames as these were the most used areas of the enclosures. We utilized one essential oil (benzoin, lavender, lemongrass - respectively) per week during the scent enrichment period of the study.

\subsection{2 | Behavioral data collection}

We collected behavioral data by instantaneous scan sampling (Altmann, 1974) of some behaviors (Table 2), as a comparable straightforward assessment of major behavioral states which may indicate the expression of significant stress-related (i.e., selfscratching, pacing) and non-stress-related (i.e., resting, sleeping, grooming, playing) behaviors, with behaviors recorded at 5 -min intervals over the duration of $6 \mathrm{~h}$ from 9 a.m. to 3 p.m., 10 days over a 3-month period. We recorded a total of $480 \mathrm{~h}$ of observations over the study period, with 50 scan samples each sampling day on each group.

\subsection{3 | Hormone sampling and measurements}

We collected fecal samples every morning before behavioral observations, whenever defecation was observed and the identity of the animal was known. In total, we collected 189 samples (red-ruffed lemurs $=25$; black howler monkeys $=56$; lar gibbons $=53$; siamangs $=16$; orangutans $=39$ ). The samples were stored in a freezer at $20^{\circ} \mathrm{C}$ right after collection. At the end of the study period, the collated samples were fully prepared by adding biological hazard labels onto each pot before being delivered using dry ice to the Department of Veterinary Medical Sciences and Animal Production Science of Bologna University for radioimmunoassay (RIA).

Cortisol concentrations were determined by RIA. All concentrations were expressed in $\mathrm{pg} / \mathrm{mg}$ of fecal matter. The extraction methodology followed the methods of Fontani et al. (2014). In brief, $5 \mathrm{ml}$ of a methanol:water (4:1 v/v) solution were added to $60 \mathrm{mg}$ (wet weight) of feces in capped-glass tube vials. The vials were then vortexed for $30 \mathrm{~min}$ using a multitube pulsing vortexer. After centrifugation at $1500 \mathrm{~g}$ for $15 \mathrm{~min}, 5 \mathrm{ml}$ ethyl ether (BDH Italia) and $0.2 \mathrm{ml} \mathrm{NaHCO} 3$ (5\%; Sigma Chemical Co.) were added to $1 \mathrm{ml}$ of 


\begin{tabular}{ll}
\hline Behavior & $\begin{array}{l}\text { Description } \\
\text { Lying or sitting while awake, with eyes open and arms down by side of } \\
\text { the body. }\end{array}$ \\
\hline $\begin{array}{l}\text { Resting } \\
\text { Lleeping }\end{array}$ & $\begin{array}{r}\text { Using fingers or mouth to pick through the coat, removing any foreign bodies } \\
\text { from a conspecific. }\end{array}$ \\
\hline Grooming & $\begin{array}{r}\text { Animal is engaging in activities such as chasing others, leaping around the } \\
\text { enclosure, and so forth, in a playful context. }\end{array}$ \\
\hline Playing & $\begin{array}{l}\text { An animal rubs their own body at a fast pace. } \\
\text { Animal walks back and forth in a distinct, unchanging pattern within the } \\
\text { enclosure. }\end{array}$ \\
\hline Pacing &
\end{tabular}

TABLE 2 Ethogram

supernatant. This preparation was vortexed for $1 \mathrm{~min}$ and centrifuged for $5 \mathrm{~min}$ at $1500 \mathrm{~g}$. The ether portion was aspirated with a pipette and evaporated under an airstream suction hood at $37^{\circ} \mathrm{C}$ The dry residue was redissolved into $0.5 \mathrm{ml}$ of $0.05 \mathrm{M}$ phosphatebuffered saline (PBS; pH 7.5).

Cortisol was assayed in the fecal samples according to the method of Tamanini et al. (1983). The parameters of the analyses were as follows: Sensitivity $3.10 \mathrm{pg} / 100 \mathrm{l}$; intra-assay variability $6.8 \%$; interassay variability $9.3 \%$; specificity (\%), cortisol 100 , corticosterone 9.5, 11.-hydroxyprogesterone 8.3, cortisone 5.3, 11. desoxycortisol 5.0, progesterone 0.6, desoxycorticosterone $0.5,20$. dihydrocortisone 0.4 , testosterone 0.3 , aldosterone 0.1 , dehydroepiandrosterone less than 0.0001, 5,-pregnenolone less than $0.0001,17^{`}$-estradiol less than 0.0001 , and cholesterol less than 0.0001 .

\subsection{Statistical analyses}

To assess the effect of scent enrichment on primate behavior and FGCs, we first generated three behavioral categories from the individual behavioral measures that we collected. More specifically, we generated (1) a resting category by adding up our data on resting and sleeping behaviors, (2) a social category by combining our data on grooming and play, and (3) a stress category by combining our data on pacing and self-scratching behaviors (we included scratching in this category as this is commonly considered an indicator of anxiety; Maestripieri et al., 1992). For each behavioral category, we ran two sets of analyses: for those species for which we collected data at individual-level (i.e., black howler monkey, orangutan and siamang) we ran linear mixed model (LMM) analysis, whereas for those species for which we collected data at group-level (i.e., red-ruffed lemur and lar gibbon) we used linear regression. For both types of analyses, we included species and enrichment condition (before vs. after exposure to the scent enrichment) as predictors, whereas the rates of resting, social, and stress-related behaviors were set as dependent variables in separate models. Finally, for the LMM analysis, we set individuals' ID as a random factor. A similar approach was run to test the effect of enrichment condition on FGCs, with the difference that the individual-level LMM analysis included data collected on black howler monkeys, orangutans, siamang, and red-ruffed lemurs but the regression model was run only on lar gibbons. For all the analyses, we ran each model twice: one with predictors entered as main effects, and one with predictors entered as interaction. Then, for each analysis, we compared Akaike's information criterion (AIC) values between the two models to find the model with the best fit (i.e., with the lowest AIC value). Finally, to estimate the effect size for the LMM models, we use the " $r 2$ " function implemented in the "performance" package in R (Nakagawa \& Schielzeth, 2013). All models met the assumptions of homogeneity of variance and normality of residuals.

\subsection{Ethics statement}

This study followed the guidelines for the care and use of captive animals in the United Kingdom, involving noninvasive methods for obtaining both behavioral data and fecal samples from the study subjects. In addition, the study was conducted in compliance with the Convention on International Trade in Endangered Species of Wild Fauna and Flora and approved by the Life Sciences Ethics committee at the University of Wolverhampton (UK) and the Ethics committees at Dudley Zoo \& Castle (UK) and Twycross Zoo (UK). We also confirm that our research work was consistent with the American Society of Primatologists' Principles for Ethical Treatment of Non-Human Primates.

\section{3 | RESULTS}

Our analyses showed that enrichment condition did not have a significant effect on resting rates for any of the species examined (Table 3). Conversely, the LMM analysis examining the effect of scent enrichment on social behavior among howler monkeys, orangutans and siamangs revealed a significant effect of enrichment condition on rates of social behavior among these species (Table 4), with eight out of the 11 subjects studied exhibiting a decrease in social behavior after the introduction of scent enrichment (Figure 1). 
TABLE 3 Results of LMM and regression analyses testing the effect of enrichment condition, and species on resting rates

\begin{tabular}{llccccc} 
Analysis & Predictors & Estimate & SE & $t$ Value & $p$ Value & $R 2$ \\
\hline LMM & Intercept & 0.42 & 0.06 & 7.04 & $<.001$ & 0.43 \\
& Species (Orangutan) & 0.02 & 0.09 & 0.25 & .812 & \\
& Species (Siamang) & -0.11 & 0.09 & -1.22 & .254 & \\
& Enrichment condition & -0.02 & 0.03 & -0.63 & .534 & \\
\multirow{2}{*}{ Regression } & Intercept & 0.33 & 0.03 & 10.00 & $<.001$ & 0.80 \\
& Species & 0.26 & 0.04 & 7.43 & $<.001$ & \\
& Enrichment condition & -0.06 & 0.03 & -1.70 & .111 & \\
\hline
\end{tabular}

Note: Significant result is shown in bold.

Abbreviations: LMM, linear mixed model analysis; SE, standard error.

\begin{tabular}{|llccccc} 
Analysis & Predictors & Estimate & SE & $t$ Value & $p$ Value & $R 2$ \\
\hline LMM & Intercept & 0.08 & 0.03 & 2.65 & .028 & 0.18 \\
& Species (Orangutan) & 0.02 & 0.04 & 0.43 & .679 & \\
& Species (Siamang) & -0.02 & 0.04 & -0.42 & .683 & \\
& Enrichment condition & 0.04 & 0.02 & 2.05 & .043 & \\
\multirow{2}{*}{ Regression } & Intercept & 0.14 & 0.02 & 9.38 & $<.001$ & 0.46 \\
& Species & -0.06 & 0.02 & -2.75 & .017 & \\
& Enrichment condition & -0.02 & 0.02 & -0.86 & .405 & \\
& Enrichment condition $\times$ Species & 0.07 & 0.03 & 2.54 & .025 & \\
\hline
\end{tabular}

Note: Significant result is shown in bold.

Abbreviations: LMM, linear mixed model analysis; SE, standard error.
TABLE 4 Results of LMM and regression analyses testing the effect of enrichment condition, and species on social rates
FIGURE 1 Mean rates \pm standard error of the mean of social behavior per individual among black howler monkey, orangutan and siamang. The linear mixed model analysis analysis revealed a significant effect of the scent enrichment on social behaviors, with eight out of the 11 subjects studied exhibiting a decrease in social interactions after the introduction of the essential oils and enrichment condition had a significant impact on rates of social behavior (Table 4). This analysis revealed that though rates of social interactions among lar gibbons were comparable between before

and after exposure to the scent enrichment, among red-ruffed
1). Similarly, the regression analysis conducted on lar gibbor and red-ruffed lemurs showed that the interaction between species 


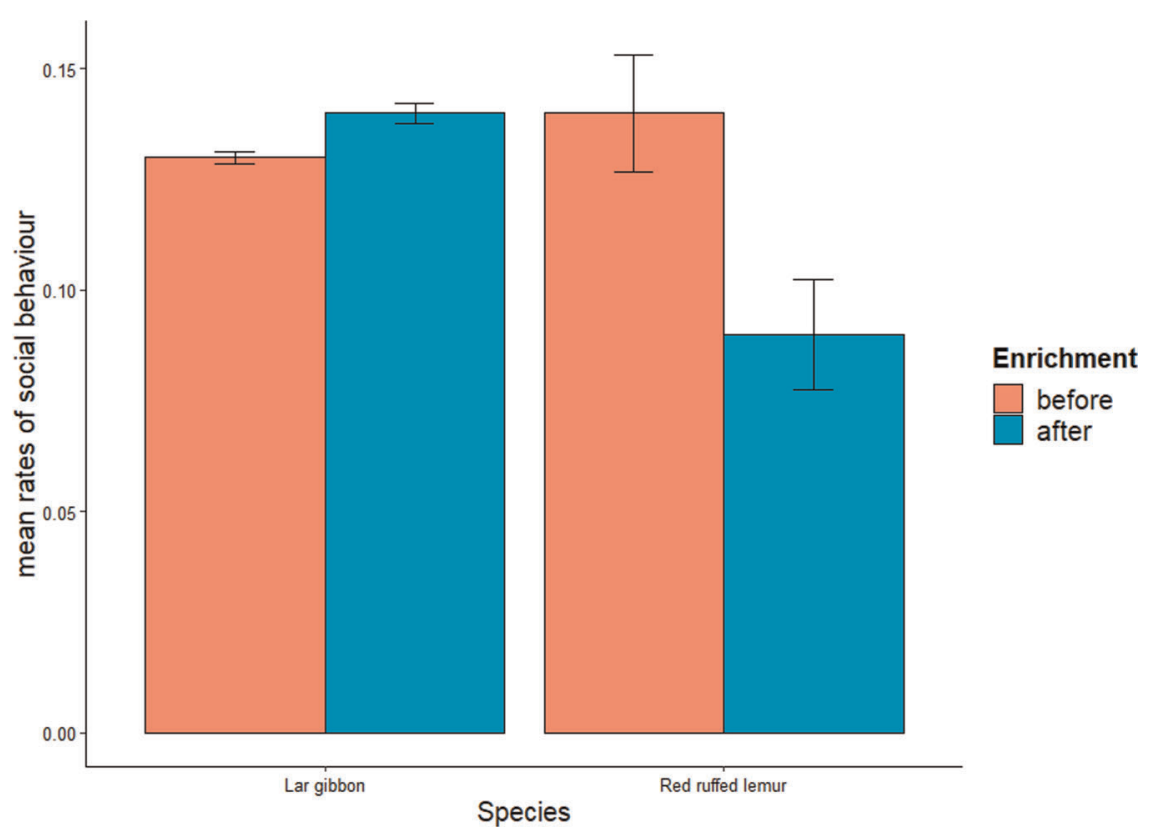

FIGURE 2 Mean rates \pm standard error of the mean of social behavior among lar gibbon and red-ruffed lemur. The regression analysis showed that the introduction of the scent enrichment induced a significant reduction in social behaviors among red-ruffed lemurs

lemurs the introduction of scent enrichment significantly reduced rates of social behavior (Figure 2).

We did not find any significant effect of enrichment condition on rates of stress-related behavior on howler monkeys, orangutans and siamangs via the LMM analysis (Table 5). By contrast, we found a significant effect of the enrichment condition on rates of stressrelated behavior among lar gibbons and red-ruffed lemurs in the regression model. This analysis showed that both species exhibited a significant reduction in rates of stress-related behavior following the exposure to scent enrichment (Figure 3).

The LMM model that investigated the effect of scent enrichment on FGCs among howler monkeys, orangutans, siamangs and red-ruffed lemurs revealed a significant interaction between species and enrichment condition (Table 6). More specifically, the analysis showed that enrichment condition affected FGCs in redruffed lemurs but not in other study species. Contrary to our expectations, however, we found that FGCs increased after exposure to scent enrichment, compared to before the introduction of the scent (Figure 4). Interestingly, Figure 4 shows that orangutans seemed to decrease their FGC levels following exposure to scent enrichment, although the effect failed to reach statistical significance. Finally, among lar gibbons, although mean FGC concentrations increased after the introduction of scent enrichment (Figure 4), the regression analysis did not reveal any significant effect of enrichment condition on FGCs (estimate $=-1.71, \mathrm{SE}=$ 1.82, $t=-0.94, p=.35, R 2=0.04)$.

\section{4 | DISCUSSION}

The effects of scent enrichment have previously been tested on several domestic, farm, laboratory, and zoo-housed animals (Blackie \& de Sousa, 2019; Heitman et al., 2018). However, olfactory stimulation is still one of the least studied forms of enrichment (reviewed in Campbell-Palmer \& Rosell, 2011). In addition, there are mixed and conflicting assumptions regarding the benefits of olfactory

\begin{tabular}{lllllll} 
Analysis & Predictors & Estimate & SE & $t$ Value & $p$ Value & $R 2$ \\
\hline \multirow{2}{*}{ LMM } & Intercept & -0.002 & 0.03 & -0.06 & 0.96 & 0.78 \\
& Species (Orangutan) & 0.003 & 0.04 & 0.09 & 0.93 & \\
& Species (Siamang) & 0.104 & 0.04 & 2.49 & 0.04 & \\
& Enrichment condition & 0.007 & 0.01 & 1.00 & 0.32 & \\
\multirow{2}{*}{ Regression } & Intercept & 0.01 & 0.01 & 1.37 & 0.192 & 0.30 \\
& Species & 0.00 & 0.01 & -0.49 & 0.633 & \\
& Enrichment condition & 0.02 & 0.01 & 2.42 & 0.030 & \\
\hline
\end{tabular}

TABLE 5 Results of LMM and regression analyses testing the effect of enrichment condition, and species on rates of stress-related behavior

Note: Significant result is shown in bold.

Abbreviations: LMM, linear mixed model analysis; SE, standard error. 
FIGURE 3 Mean rates \pm standard error of the mean of stress-related behaviors among lar gibbon and red-ruffed lemur. The regression model showed that both lar gibbon and red-ruffed lemur exhibited a significant reduction in stress-related behaviors following the exposure to the scent enrichment

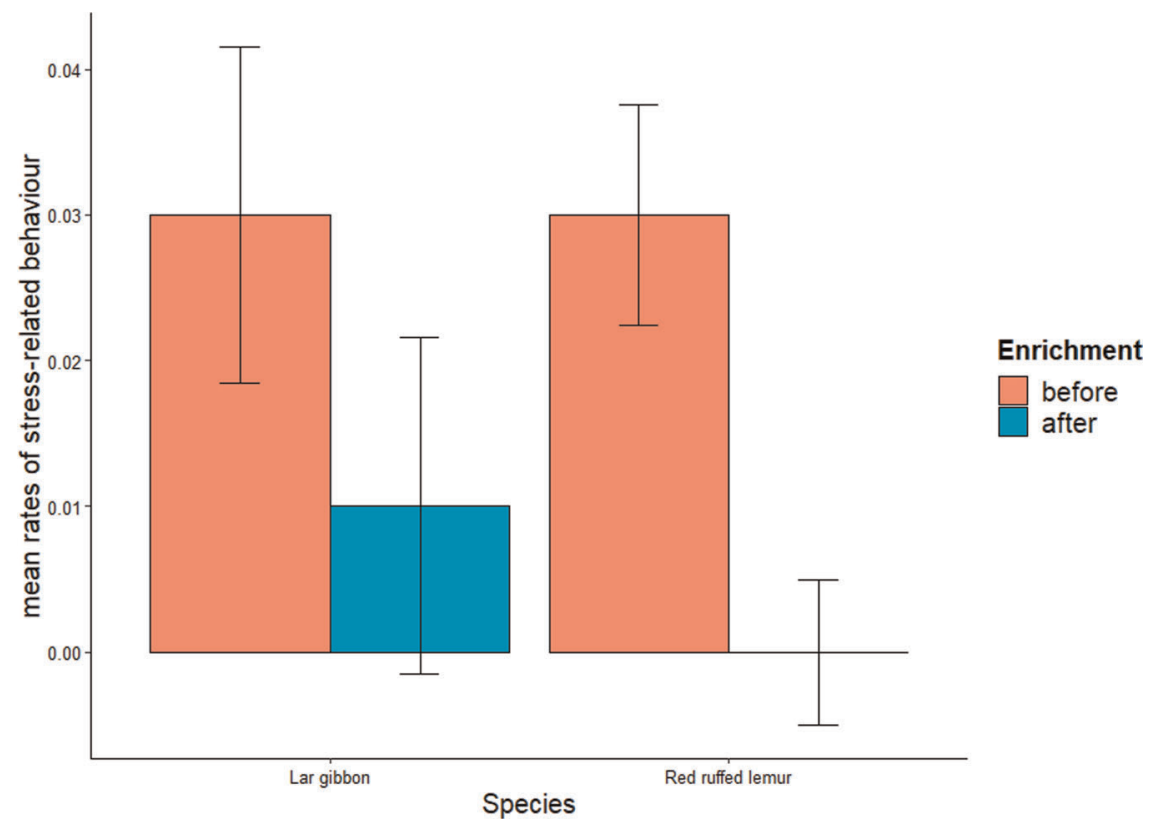

enrichment on animal welfare and this is particularly the case of primate species, among which the overall effects of scent enrichment are still unclear and understudied (reviewed in Wells, 2009).

Unexpectedly, we found a significant reduction in rates of social interactions after being exposed to the series of essential oils in both red-ruffed lemurs and black howler monkeys. By contrast, several authors have found that scent enrichment may cause increasing active behaviors in zoo-housed non-primate species, such as cheetahs (Acinonyx jubatus) (Quirke \& O'Riordan, 2011), Californian sea lions (Zalophus californianus) (Samuelson et al., 2017), and Rothschild giraffes (Giraffa camelopardalis rothschildi) (Fay \& Miller, 2015), but not in meerkats (Suricata suricatta) (Myles \& Montrose, 2015). Regarding primates, Gronqvist et al. (2013) showed that olfactory enrichment significantly increased the frequency of natural speciesspecific behaviors in captive Javan gibbons (Hylobates moloch) although the interest in the new scent decreased rapidly after the first day, though no significant effects on individual behaviors were found in ring-tailed lemurs (Lemur catta) (Baker et al., 2018) and gorillas (Gorilla gorilla gorilla) (Wells et al., 2007). The effect that our scent enrichment exerted on social behaviors, with decreased rates of social interactions in red-ruffed lemurs and black howler monkeys, but no significant effects on siamangs, lar gibbons, and orangutans, might be related to differences in social organizations and structures among these species. Specifically, red-ruffed lemurs and black howler monkeys are social species living in small groups including both adult males and females, whereas siamangs and lar gibbons are monogamous and orangutans are solitary. Red-ruffed lemurs and black howler monkeys, thus, display more social affiliative behaviors which have a stress-reducing effect. We, therefore, speculate that redruffed lemurs and black howler monkeys could have reduced their rates of social behaviors because our scent enrichment might have decreased the need for reassurance-derived social interactions.
TABLE 6 Results of linear mixed model (LMM) analysis testing the effect of enrichment condition, and species on fecal glucocorticoid concentrations

$\begin{array}{llccccc}\text { Analysis } & \text { Predictors } & \text { Estimate } & \text { SE } & t \text { Value } & p \text { Value } & R 2 \\ \text { LMM } & \text { Intercept } & 0.91 & 0.40 & 2.28 & 0.028 & 0.28 \\ & \text { Species (Orangutan) } & -0.46 & 0.65 & -0.70 & 0.491 & \\ \text { Species (Red-ruffed lemur) } & 1.30 & 0.84 & 1.55 & 0.130 & \\ \text { Species (Siamang) } & -0.87 & 0.76 & -1.14 & 0.263 & \\ \text { Enrichment condition } & 0.27 & 0.59 & 0.46 & 0.648 & \\ \quad \begin{array}{l}\text { Enrichment condition } \times \text { species } \\ \quad \text { (Orangutan) }\end{array} & 1.25 & 0.83 & 1.49 & 0.141 & \\ \quad \text { Enrichment condition } \times \text { species (Red- } & -2.04 & 0.95 & -2.16 & 0.035 & \\ \quad \text { ruffed lemur) } & & & & & & \\ \quad \text { Enrichment condition } \times \text { species (Siamang) } & -0.26 & 1.27 & -0.21 & 0.838 & \end{array}$

Note: The bold values indicate statistically significant interaction $(\mathrm{p}<0.05)$ between a study species (red-ruffed lemur) and the enrichment condition. 


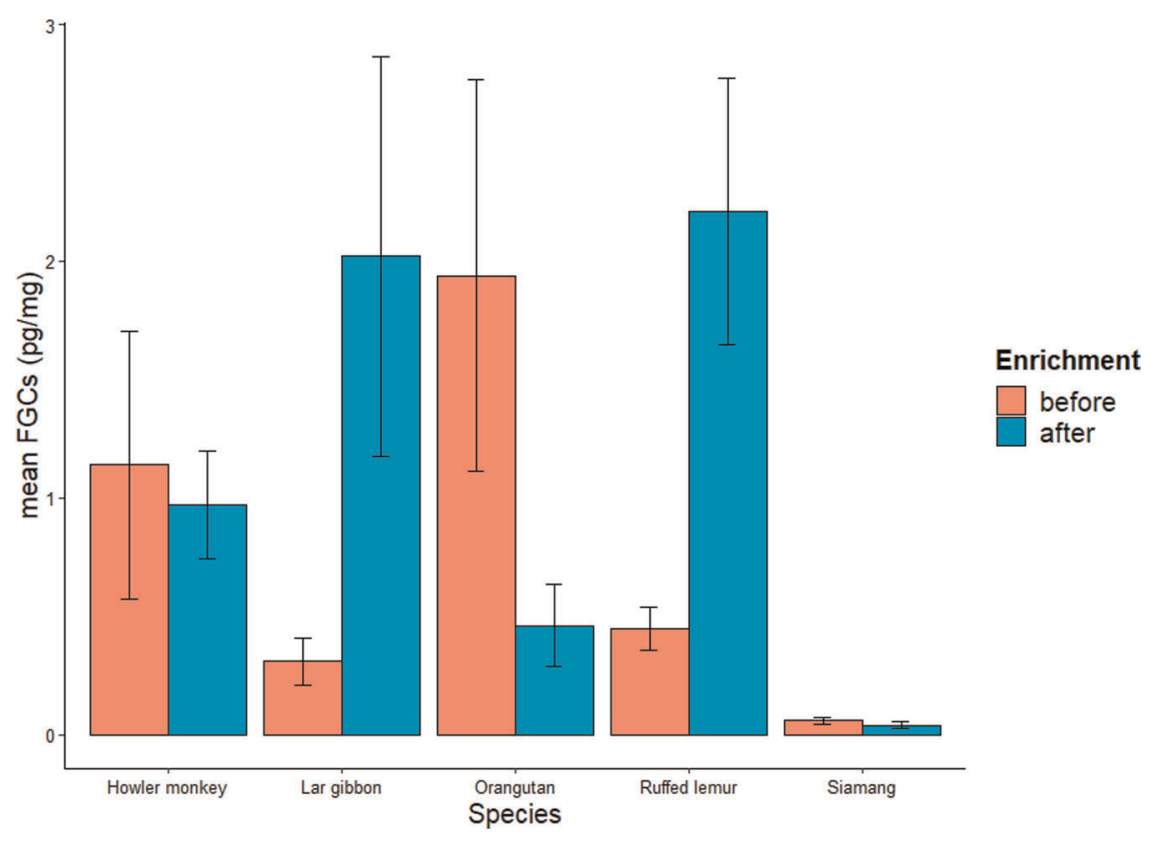

FIGURE 4 The linear mixed model showed that the scent enrichment elicited an increase in fecal glucocorticoid concentration levels in red-ruffed lemurs, but not in any other study species.
However, we recognize that further factors may have induced such differences between the effects of our scent enrichment on individual species; for instance, it is possible that the new unfamiliar scents increased the stress levels in red-ruffed lemurs because they perceived them more intensely than the other study species, or that decreased rates in social behaviors in red-ruffed lemurs and black howler monkeys are due to increased rates in other behaviors (such as inspections, locomotion, etc.) which were not measured during our study.

We also found a significant reduction in rates of stress-related behaviors after red-ruffed lemurs and lar gibbons were exposed to the series of essential oils, which is the most promising outcome of this preliminary study about the potential positive effect of such essential oils. Similar findings have been reported in non-primate species. For example, Uccheddu et al. (2018) exposed domestic dogs to a variety of essentials oils and found that some scents increased frequencies of behavioral indicators of relaxation, whereas others decreased behavioral indicators of stress, such as pacing and overgrooming. Similarly, a study on cheetahs and Sumatran tigers (Panthera tigris sumatrae) found that stereotypic pacing behavior significantly decreased in the presence of a hay ball with cinnamon (Damasceno et al., 2017). The significant effect from our series of essential oils on stress-related behaviors in red-ruffed lemurs and lar gibbons is consistent with our prediction that scent enrichment would reduce behavioral indicators of stress; however, we acknowledge the conflicting findings related to the lack of effectiveness of our enrichment shown in siamangs, lar gibbons, and orangutans.

Our finding that red-ruffed lemurs showed a significant increase in FGC levels following the exposure to the series of essential oils may suggest different interpretations. First, this result implies that changes in behavioral indicators of stress, such as pacing and selfscratching, do not necessarily mirror changes in physiological indicators of stress, such as FGCs. This is consistent with the study by Higham et al. (2009) on olive baboons (Papio anubis) showing that day-to-day variation in FGC concentrations do not correlate with changes in self-directed behaviors and suggesting that these indicators may signpost two different types of stress, with self-directed behaviors reflecting low-level acute stress or anxiety (Maestripieri et al., 1992) but FGCs reflecting high-level chronic stress (Sapolsky, 2002). Accordingly, self-directed behaviors have been found to increase in anxiety-inducing contexts, such as when animals are given anxiogenic drugs (Schino et al., 1996) or after aggression (Schino, 1998); FGC concentrations have been shown to increase when animals are exposed to high levels of stress, such as in the presence of tourists (Barja et al., 2007) or when exposed to the odor of a predator (Monclús et al., 2006). Additionally, although glucocorticoids are commonly associated with the negative aspects of stress, these steroid hormones play many important roles both in mediating the response to stress and in the circadian rhythm (McEwen, 2019). Thus, another potential explanation for elevated FGCs is increased energy expenditure. For instance, it is possible that increased FGCs in red-ruffed lemurs may be due to enhanced positive arousal related to increased rates in other behaviors (such as investigatory behaviors and locomotion) which we did not measure during our study. Hence, as suggested by other authors (reviewed by G. Hosey et al., 2013), we emphasize that both behavioral and physiological indicators should be used to investigate the stress levels of individual animals, whereas behavioral indicators of anxiety alone should not be interpreted as definite indicators of glucocorticoid production. Interestingly, we found that our scent enrichment exerted both behavioral and physiological effects only on red-ruffed lemurs. Although primates have traditionally been considered to be "microsmatic" with a simultaneous amplified emphasis on vision (Dominy \& Lucas, 2001; Fornalé et al., 2012; Gerald, 2003), several studies suggest that chemical communication is important also for 
primate species (reviewed by Drea, 2020). Particularly, it is established that some species rely heavily on olfaction in addition to vision and auditory senses; for instance, this is the case of several lemurs (Gould \& Overdorff, 2002; Janda et al., 2019; Scordato \& Drea, 2007) and squirrel monkeys (Laska et al., 2000). This would explain the significant impact of our scent enrichment on red-ruffed lemurs, rather than the other study species for which no such response was observed, as lemurs have retained a greater olfactory complexity than other lineages such as monkeys and apes. However, we recognize that other factors may have contributed to such effects of our scent enrichment on individual species; for instance, it is possible that the effect on red-ruffed lemurs could be related to their different enclosure design (i.e., "visitor walkthrough"-including a section in which the public could be very close) which ultimately could have led the lemurs being exposed to a different olfactory environment (i.e., anthropogenic) than the other study species.

Finally, we have to acknowledge some major limitations of this preliminary study. First of all, although our study is ambitious in many respects (i.e., we worked on several species, over several years, across three conditions and with multiple measures intended to assess welfare), we focused on limited data pools which included a relatively small sample size and unit of analysis. Additionally, we did not record behaviors, such as normal locomotion, foraging, inspections and investigatory actions (e.g., exploring around the scented cloths), but changes in these behaviors could also be very informative.

\section{5 | CONCLUSION}

This preliminary study provided contradictory findings and suggested that the application of our new scent enrichment program may affect the stress levels of zoo-housed primates; particularly in the case of primate species where odor plays a crucial role, such as red-ruffed lemurs. Following the exposure to the series of essential oils (benzoin, lavender, and lemongrass), both red-ruffed lemurs and lar gibbons exhibited significantly lower rates of stress-related behaviors, such as pacing and self-scratching. Conversely, red-ruffed lemurs also significantly increased their levels of FGCs, which however might be explained by an increase in positive arousal. However, given that the exposure to the series of essential oils entailed a significant reduction in social behaviors in red-ruffed lemurs and black howler monkeys as well as a significant increase in FGCs in redruffed lemurs, we cannot even exclude negative effects by our scent enrichment. Therefore, in conclusion, we cannot recommend using this series of essential oils widely without further evaluation.

Future work would need to expand the investigation of the effect of our scent enrichment on primate welfare by focusing on both a larger sample size and a wider range of species across the major lineages. Also, it would be crucial to test further types of scent enrichment by considering the ecological/biological relevance of the scent enrichment to the study species. Many scents, including essential oils, are chosen based on their effectiveness in humans or domestic animals, but this may not necessarily be appropriate for all animal species (Wells, 2009). In particular, as previous authors have suggested, important factors to consider for the implementation of novel olfactory enrichment programs are the mechanism of delivery of the scent and the type of scents used (Baker et al., 2018), whereas the effectiveness of any intervention should be continually monitored to inform best practices.

\section{ACKNOWLEDGMENTS}

We are grateful to Dudley Zoo \& Castle (especially David Beeston, Chris Leeson, Pat Stevens, and primate keepers) and Twycross Zoo (especially Mat Liptovszky, Manuela Townsend, Freisha Patel, Jessica Rendle, and primate keepers) for their support to the project and assistance with sample collection. We thank Jemma Billingsley, Stephanie Courten and Henry Swain for collecting additional data which provided helpful insight but could not be included in this study. Finally, we thank two anonymous reviewers for their constructive comments and suggestions. This study work was supported by the Faculty of Science and Engineering, University of Wolverhampton (equipment \& laboratory consumables), and the Department of $\mathrm{Ve}-$ terinary Medical Sciences, University of Bologna (laboratory analyses). This project also received funding from the Primate Society of Great Britain (Captive Care Grant-round 2018 to Stefano Vaglio).

\section{CONFLICT OF INTERESTS}

The authors declare that there are no conflict of interests.

\section{AUTHOR CONTRIBUTIONS}

Stefano Vaglio: Conceptualization (lead); funding acquisition (lead); investigation (lead); methodology (equal); project administration (lead); resources (equal); supervision (lead); writing original draft (lead); writing review \& editing (lead). Richard Pearce: Data curation (lead); formal analysis (supporting); resources (equal); supervision (supporting). Luke Bryant: Conceptualization (supporting); data curation (supporting); investigation (supporting). Alexandria Lott: Conceptualization (supporting); data curation (supporting); investigation (supporting). Ailie McAuley: Conceptualization (supporting); data curation (supporting); investigation (supporting). Demi J. Sheppard: Conceptualization (supporting); data curation (supporting); investigation (supporting). Sarah Smith: Conceptualization (supporting); data curation (equal); investigation (equal). Beth Tompkins: conceptualization (supporting); data curation (supporting); investigation (supporting). Emily Elwell: Conceptualization (supporting); writing original draft (supporting). Sara Fontani: Conceptualization (supporting); writing original draft (supporting). Christopher Young: Supervision (supporting); writing original draft (supporting). Giovanna Marliani: Investigation (supporting); methodology (equal). Pier A. Accorsi: Conceptualization (supporting); funding acquisition (supporting); methodology (equal); resources (equal); supervision (supporting).

\section{PEER REVIEW}

The peer review history for this article is available at https://publons. com/publon/10.1002/ajp.23247. 


\section{DATA AVAILABILITY STATEMENT}

The raw data are available on request.

\section{ORCID}

Stefano Vaglio (D) https://orcid.org/0000-0003-0885-8573

Stefano S. K. Kaburu (D) https://orcid.org/0000-0001-7456-3269

Sara Fontani (D) https://orcid.org/0000-0002-9338-8555

Christopher H. Young (D) https://orcid.org/0000-0001-6090-7315

Pier Attilio Accorsi (D) https://orcid.org/0000-0001-8614-7131

\section{REFERENCES}

Altmann, J. (1974). Observational study of behaviour: Sampling methods. Behaviour, 49, 227-267. https://doi.org/10.1163/156853974X00534

Baker, B., Taylor, S., \& Montrose, V. T. (2018). The effects of olfactory stimulation on the behavior of captive ring-tailed lemurs (Lemur catta). Zoo Biology, 37, 16-22. https://doi.org/10.1002/zoo.21392

Barja, I., Silván, G., Rosellini, S., Piñeiro, A., González-Gil, A., Camacho, L., \& Illera, J. C. (2007). Stress physiological responses to tourist pressure in a wild population of European pine marten. The Journal of Steroid Biochemistry and Molecular Biology, 104, 136-142. https://doi.org/10. 1016/j.jsbmb.2007.03.008

Ben-Ari, E. T. (2001). What's new at the zoo? Zoo biologists are taking a scientific approach to improving the quality of life for captive animals. BioScience, 51, 172-177. https://doi.org/10.1641/0006-3568

Blackie, N., \& de Sousa, M. (2019). The use of garlic oil for olfactory enrichment increases the use of ropes in weaned pigs. Animals: An Open Access Journal from MDPI, 9, 148. https://doi.org/10.3390/ ani9040148

Campbell-Palmer, R., \& Rosell, F. (2011). The importance of chemical communication studies to mammalian conservation biology: A review. Biological Conservation, 144, 1919-1930. https://doi.org/ 10.1016/j.biocon.2011.04.028

Carlstead, K., \& Shepherdson, D. (1994). Effects of environmental enrichment on reproduction. Zoo Biology, 13, 447-458. https://doi. org/10.1002/zoo.1430130507

Carroll, S.P., Jorgensen, P. S., Kinnison, M. T., Bergstrom, C. T., Denison, R.F., Gluckman, P., Smith, T. B., Strauss, S. Y., \& Tabashnik, B.E. (2014). Applying evolutionary biology to address global challenges. Science, 346(6207), 1245993. https://doi.org/10. 1126/science.1245993

Clark, F., \& King, A. J. (2008). A critical review of zoo-based olfactory enrichment. In J. L. Hurst, R. J. Benyon, S. C. Roberts, \& T.D. Wyatt (Eds.), Chemical Signals in Vertebrates 11 (pp. 391-398). Springer.

Damasceno, J., Genaro, G., Quirke, T., McCarthy, S., McKeown, S., \& O'Riordan, R. (2017). The effects of intrinsic enrichment on captive felids. Zoo Biology, 36, 186-192. https://doi.org/10.1002/zoo.21361

Danial Rioldi, E. (2013). The influence of conservation breeding programs on animal communication and behavior-A literary review (Unpublished Master's thesis). Mälardalen University, Västerås, Sweden.

Dominy, N. J., \& Lucas, P. W. (2001). Ecological importance of trichromatic vision to primates. Nature, 410, 363-366. https://doi. org/10.1038/35066567

Drea, C. M. (2020). Design, delivery and perception of conditiondependent chemical signals in strepsirrhine primates: Implications for human olfactory communication. Philosophical Transactions of the Royal Society B, 375, 20190264. https://doi.org/10.1098/rstb. 2019.0264

Ellis, S. L. H., \& Wells, D. L. (2010). The influence of olfactory stimulation on the behaviour of cats housed in a rescue shelter. Applied Animal Behaviour Science, 123, 56-62. https://doi.org/10.1016/j.applanim. 2009.12.011
Estrada, A., Garber, P. A., Rylands, A. B., Roos, C., Fernandez-Duque, E., Di Fiore, A., Nekarisi, K. A. I., Nijman, V., Heymann, E. W., Lambert, J. E., Rovero, F., Barelli, C., Setchell, J. M., Gillespie, T. R., Mittermeier, R. A., Arregoitia, L. V., de Guinea, M., Gouveia, S., Dobrovolski, R., ... Li, B. (2017). Impending extinction crisis of the world's primates: Why primates matter. Science Advances, 3, e1600946. https://doi.org/10.1126/sciadv.1600946

Fay, C., \& Miller, L. (2015). Utilizing scents as environmental enrichment: Preference assessment and application with Rothschild giraffe. Animal Behavior \& Cognition, 2, 285-291. https://doi.org/10.12966/ abc.08.07.2015

Fontani, S., Vaglio, S., Beghelli, V., Mattioli, M., Bacci, S., \& Accorsi, P. A. (2014). Fecal concentrations of cortisol, testosterone, and progesterone in cotton-top tamarins housed in different zoological parks: Relationships among physiological data, environmental conditions, and behavioral patterns. Journal of Applied Animal Welfare Science, 17, 228-252. https:// doi.org/10.1080/10888705.2014.916173

Fornalé, F., Vaglio, S., Spiezio, C., \& Prato Previde, E. (2012). Red-green colour vision in three catarrhine primates. Communicative Integrative Biology, 5, 583-589. https://doi.org/10.4161/cib.21414

Gerald, M. S. (2003). How color may guide the primate world: Possible relationships between sexual selection and sexual dichromatism in sexual selection and reproductive competition in primates. In C. B. Jones (Ed.), New Perspectives and Directions. American Society of Primatologists. Special Topics in Primatology.

Gould, L., \& Overdorff, D. (2002). Adult male scent-marking in Lemur catta and Eulemur fulvus rufus. International Journal of Primatology, 23, 575-596. https://doi.org/10.1023/A:1014921701106

Gronqvist, G., Kingston-Jones, M., May, A., \& Lehmann, J. (2013). The effects of three types of environmental enrichment on the behaviour of captive Javan gibbons (Hylobates moloch). Applied Animal Behaviour Science, 147, 214-223. https://doi.org/10.1016/j. applanim.2013.04.021

Heitman, K., Rabquer, B., Heitman, E., Streu, C., \& Anderson, P. (2018). The use of lavender aromatherapy to relieve stress in trailered horses. Journal of Equine Veterinary Science, 63, 8-12. https://doi.org/ 10.1016/j.jevs.2017.12.008

Higham, J. P., MacLarnon, A. M., Heistermann, M., Ross, C., \& Semple, S. (2009). Rates of self-directed behaviour and faecal glucocorticoid levels are not correlated in female wild olive baboons (Papio hamadryas anubis). Stress (Amsterdam), 12, 526-532. https://doi.org/ 10.3109/10253890902756565

Holland, C. (2018). Olfactory cognitive enrichment training for a male Asian elephant. Gajah, 48, 34-37.

Horvath, J. L., Croswell, M., O'Malley, R. C., \& McGrew, W. C. (2007). Plant species with potential as food, nesting material, or tools at a chimpanzee refuge site in Caddo Parish, Louisiana. International Journal of Primatology, 28, 135-158. https://doi.org/10.1007/ s10764-006-9106-4

Hosey, G., Melfi, V., \& Pankhurst, S. (2013). Zoo animals: Behaviour, Management, and Welfare. Oxford University Press.

Hosey, G. R. (2005). How does the zoo environment affect the behaviour of captive primates? Applied Animal Behaviour Science, 90, 107-129. https://doi.org/10.1016/j.applanim.2004.08.015

International Union for Conservation of Nature (IUCN). (2020). The IUCN Red List of Threatened Species.

Janda, E. D., Perry, K. L., Hankinson, E., Walker, D., \& Vaglio, S. (2019). Sex differences in scent-marking in captive red-ruffed lemurs. American Journal of Primatology, 81, e22951. https://doi.org/10.1002/ajp.22951

Laska, M., Seibt, A., \& Weber, A. (2000). 'Microsmatic' primates revisited: Olfactory sensitivity in the squirrel monkey. Chemical Senses, 25, 47-53. https://doi.org/10.1093/chemse/25.1.47

Maestripieri, D., Schino, G., Aureli, F., \& Troisi, A. (1992). A modest proposal: Displacement activities as an indicator of emotions in 
primates. Animal Behaviour, 44, 967-979. https://doi.org/10.1016/ S0003-3472(05)80592-5

McEwen, B. C. (2019). What is the confusion with cortisol? Chronic Stress, 3, 2470547019833647. https://doi.org/10.1177/2470547019833647

Meier, K. (2016). Blue eyes on red lists: Conservation and the future of the blue-eyed black lemur (Unpublished award-winning anthropology papers). Macalester College, Saint Paul, MN.

Mellor, D. J., Hunt, S., \& Gusset, M. (2015). Caring for wildlife: The world zoo and aquarium animal welfare strategy. WAZA Executive Office.

Monclús, R., Rödel, H. G., Palme, R., von Holst, D., \& de Miguel, J. (2006). Non-invasive measurement of the physiological stress response of wild rabbits to the odour of a predator. Chemoecology, 16, 25-29. https://doi.org/10.1007/s00049-005-0324-6

Myles, S., \& Montrose, V. T. (2015). The effects of olfactory stimulation on the behaviour of captive meerkats (Suricata suricatta). Journal of Zoo and Aquarium Research: A Journal of Science and its Applications, 3, 37-42. https://doi.org/10.19227/jzar.v3i2.97

Nakagawa, S., \& Schielzeth, H. (2013). A general and simple method for obtaining R2 from generalized linear mixed-effects models. Methods in Ecology \& Evolution, 4, 133-142. https://doi.org/10.1111/j.2041210x.2012.00261.x

Negus, V. (1958). The comparative anatomy and physiology of the nose and paranasal sinuses. E \& S Livingstone.

Norland, D. A., \& Lewis, W. J. (1976). Semiochemicals. John Wiley and Sons.

Quirke, T., \& O'Riordan, R. M. (2011). The effect of a randomised enrichment treatment schedule on the behaviour of cheetahs (Acinonyx jubatus). Applied Animal Behaviour Science, 135, 103-109. https://doi.org/10.1016/j.applanim.2011.10.006

Rafacz, M. L., \& Santymire, R. M. (2014). Using odor cues to elicit a behavioral and hormonal response in zoo-housed African wild dogs. Zoo Biology, 33, 144-149. https://doi.org/10.1002/zoo.21107

Samuelson, M. M., Lauderdale, L. K., Pulis, K., Solangi, M., Hoffland, T., \& Lyn, H. (2017). Olfactory enrichment in California sea lions (Zalophus californianus): An effective tool for captive welfare? Journal of Applied Animal Welfare Science, 20, 75-85. https://doi. org/10.1080/10888705.2016.1246362

Sapolsky, R. M. (2002). Endocrinology of the stress-response. In J. B. Becker, S. M. Breedlove, D. Crews, \& M. M. McCarthy (Eds.), Behavioral endocrinology (pp. 408-450). The MIT Press.

Schino, G. (1998). Reconciliation in domestic goats. Behaviour, 135, 343-356. https://doi.org/10.1163/156853998793066302

Schino, G., Perretta, G., Taglioni, A. M., Monaco, V., \& Troisi, A. (1996). Primate displacement activities as an ethopharmacological model of anxiety. Anxiety, 2, 186-191. https://doi.org/10.1002/(SICI)15227154(1996)2:4<186::AID-ANXI5>3.0.CO;2-M

Schulte-Hostedde, A. I., \& Mastromonaco, G. F. (2015). Integrating evolution in the management of captive zoo populations.
Evolutionary Applications, 8, 413-422. https://doi.org/10.1111/eva. 12258

Scordato, E. S., \& Drea, C. (2007). Scents and sensibility: Information content of olfactory signals in the ring-tailed lemur, Lemur catta. Animal Behaviour, 73, 301-314. https://doi.org/10.1016/j.anbehav.2006.08.006

Setchell, J. M., Vaglio, S., Moggi-Cecchi, J., Boscaro, F., Calamai, L., \& Knapp, L. A. (2010). Chemical composition of scent-gland secretions in an Old World monkey (Mandrillus sphinx): Influence of sex, male status, and individual identity. Chemical Senses, 35, 205-220. https:// doi.org/10.1093/chemse/bjp105

Smith, T. D., \& Bhatnagar, K. P. (2004). Microsmatic primates: Reconsidering how and when size matters. Anatomical Record. Part B, New Anatomist, 279, 24-31. https://doi.org/10.1002/ar.b.20026

Tamanini, C., Giordano, N., Chiesa, F., \& Seren, E. (1983). Plasma cortisol variations induced in the stallion by mating. European Journal of Endocrinology, 102, 447-450. https://doi.org/10.1530/acta.0.1020447

Uccheddu, S., Mariti, M., Sannen, A., Vervaecke, H., Arnout, H., Gutierrez, R. J., Haverbeke, A., \& Gazzano, A. (2018). Behavioural and cortisol responses of shelter dogs to a cognitive bias test after olfactory enrichment with essential oils. Dog Behavior, 2, 1-14. https://doi.org/10.4454/db.v4i2.87

Vaglio, S., Minicozzi, P., Romoli, R., Boscaro, F., Pieraccini, G., Moneti, G., \& Moggi-Cecchi, J. (2016). Sternal gland scent-marking signals sex, age, rank, and group identity in captive mandrills. Chemical Senses, 41, 177-186. https://doi.org/10.1093/chemse/bjv077

Wells, D. L. (2004). A review of environmental enrichment for kennelled dogs, Canis familiaris. Applied Animal Behaviour Science, 85, 307-317. https://doi.org/10.1016/j.applanim.2003.11.005

Wells, D. L. (2009). Sensory stimulation as environmental enrichment for captive animals: A review. Applied Animal Behaviour Science, 118, 1-11. https://doi.org/10.1016/j.applanim.2009.01.002

Wells, D. L., Hepper, P. G., Coleman, D., \& Challis, M. G. (2007). A note on the effect of olfactory stimulation on the behaviour and welfare of zoo-housed gorillas. Applied Animal Behaviour Science, 106, 155-160. https://doi.org/10.1016/j.applanim.2006.07.010

How to cite this article: Vaglio, S., Kaburu, S. S. K., Pearce, R., Bryant, L., McAuley, A., Lott, A., Sheppard, D. J., Smith, S., Tompkins, B., Elwell, E., Fontani, S., Young, C., Marliani, G., \& Accorsi, P. A. (2021). Effects of scent enrichment on behavioural and physiological indicators of stress in zoo primates. American Journal of Primatology, e23247.

https://doi.org/10.1002/ajp.23247 